ARTICLE

https://doi.org/10.1038/s41467-019-09706-9

\title{
Inner-shell clock transition in atomic thulium with a small blackbody radiation shift
}

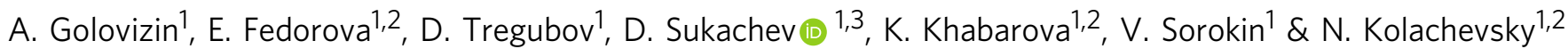

One of the key systematic effects limiting the performance of state-of-the-art optical clocks is the blackbody radiation (BBR) shift. Here, we demonstrate unusually low sensitivity of a 1.14 $\mu \mathrm{m}$ inner-shell clock transition in neutral Tm atoms to BBR. By direct polarizability measurements, we infer a differential polarizability of the clock levels of -0.063(30) atomic units corresponding to a fractional frequency BBR shift of only $2.3(1.1) \times 10^{-18}$ at room temperature. This amount is several orders of magnitude smaller than that of the best optical clocks using neutral atoms $(\mathrm{Sr}, \mathrm{Yb}, \mathrm{Hg})$ and is competitive with that of ion optical clocks $\left(\mathrm{Al}^{+}, \mathrm{Lu}^{+}\right)$. Our results allow the development of lanthanide-based optical clocks with a relative uncertainty at the $10^{-17}$ level.

\footnotetext{
${ }^{1}$ P.N. Lebedev Physical Institute, Leninsky prospekt 53, Moscow 119991, Russia. 2 Russian Quantum Center, Business-Center "Ural", $100 A$ Novaya St. SkolkovoMoscow 143025, Russia. ${ }^{3}$ Physics Department of Harvard University, 17 Oxford Str., Cambridge, MA 02138, USA. Correspondence and requests for materials should be addressed to N.K. (email: kolachevsky@lebedev.ru)
} 
T he unprecedented performance of state-of-the-art optical atomic clocks ${ }^{1-3}$, together with the increasing number of characterized clock transitions in different atomic and ionic species, has pushed the frontiers of physics enabling sensitive tests of special relativity ${ }^{4,5}$, the search for variations of fundamental constants ${ }^{6,7}$ and dark matter ${ }^{8,9}$, atom-based quantum simulations ${ }^{10}$, and others as summarized in reviews ${ }^{11-13}$. Moreover, the development of robust, compact, and transportable optical clocks ${ }^{14}$, overcoming in accuracy the best microwave frequency standards, promises big progress in precision spectroscopy, navigation, and geodesy 15,16 .

The fractional frequency uncertainty of optical clocks reaching a low level of $10^{-18}$ was successfully demonstrated for $\mathrm{Sr}^{17}$ and $\mathrm{Yb}^{18}$ optical lattice clocks and for $\mathrm{Yb}^{+19}$ and $\mathrm{Al}^{+20}$ single-ion optical clocks. One of the current limitations is the impact of electric fields, such as surrounding blackbody radiation (BBR), trapping fields, and collisions. To extend the current limit in frequency stability and accuracy, there is a continuous search for new species, particularly with reduced sensitivity to external electric fields. Among the promising candidates are single $\mathrm{Lu}^{+}$ ions $^{21}$, highly charged ions ${ }^{22-24}$, and ${ }^{229} \mathrm{Th}$ with an isomeric nuclear transition ${ }^{25,26}$.

Lanthanides with a submerged electronic $f$-shell possess naturally suppressed sensitivity to external electric fields for innershell $f-f$ transitions because of strong shielding from the closed $5 s^{2}$ and $6 s^{2}$ shells. As early as 1984, E. Alexandrov and coauthors demonstrated unusually low spectral broadening of the $1.14 \mu \mathrm{m}$ inner-shell transition in atomic Tm under collisions with buffer

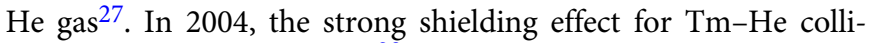
sions was confirmed in ref. ${ }^{28}$. Similar effects were also observed for some transition elements with nonzero orbital angular momentum ${ }^{29}$. For lanthanide ions doped in solids, the shielding effect reduces inhomogeneous broadening of the inner-shell $f-f$ transitions, which allows, for example, ensemble-based solid-state quantum memory ${ }^{30}$ and an integrated single-photon source in the telecom wavelength range ${ }^{31}$. However, the possibility to use such transitions for optical clocks was studied only theoretically ${ }^{32-34}$.

In this Article, we report on precision spectroscopy of the inner-shell clock transition $\left|J=7 / 2, F=4, m_{\mathrm{F}}=0\right\rangle \rightarrow \mid J=5 / 2$, $\left.F=3, \quad m_{\mathrm{F}}=0\right\rangle$ between the fine-structure components of the ground electronic state in the single stable isotope ${ }^{169} \mathrm{Tm}$ (the nuclear spin equals $I=1 / 2$ ) at a wavelength of $1.14 \mu \mathrm{m}$ with the natural linewidth of $\gamma=1.2 \mathrm{~Hz}$ (here, $J$ and $F$ stand for the electronic and total momentum quantum numbers, respectively, and $m_{\mathrm{F}}$ is the magnetic quantum number). Spectroscopy of the $\left.m_{\mathrm{F}}=0\right\rangle \rightarrow\left|m_{\mathrm{F}}=0\right\rangle$ clock transition leads to zero first-order Zeeman and magnetic dipole-dipole interaction shifts ${ }^{34}$. We experimentally determined the magic wavelength of the optical lattice near $813 \mathrm{~nm}$, recorded the Fourier-limited spectral linewidth of the clock transition of $10 \mathrm{~Hz}$ and characterized its sensitivity to electric and magnetic fields. Accurate measurement of the differential dynamic polarizabilities of clock levels in the nearinfrared spectral region (810-860 and $1064 \mathrm{~nm}$ ) allowed an estimate for the BBR frequency shift of the clock transition, which was found to be a few orders of magnitude smaller than that of other characterized clock transitions in neutral atoms.

\section{Results}

Experimental setup. Thulium atoms are laser cooled in a twostage magneto-optical trap (see Fig. 1 and the detailed description in ref. ${ }^{35}$ ). For precision spectroscopy, atoms at a temperature of $\sim 10 \mu \mathrm{K}$ are loaded in a vertical optical lattice formed inside an enhancement cavity (finesse of 20 ) with intracavity polarization filtering. This configuration allows for $6 \mathrm{~W}$ of circulating power in the cavity from a $1 \mathrm{~W}$ Ti:sapphire laser between 810 and $860 \mathrm{~nm}$. The beam waist radius can be varied between 80 and $160 \mu \mathrm{m}$ by controlling the distance between cavity elements.

We prepare atoms in lower clock state $\left|F=4, m_{\mathrm{F}}=0\right\rangle$ by driving simultaneously $|F=4\rangle \rightarrow|F=4\rangle$ (pump) and $|F=3\rangle \rightarrow$ $|F=4\rangle$ (repump) transitions with $5 \mathrm{~ms}$-long $\pi$-polarized laser
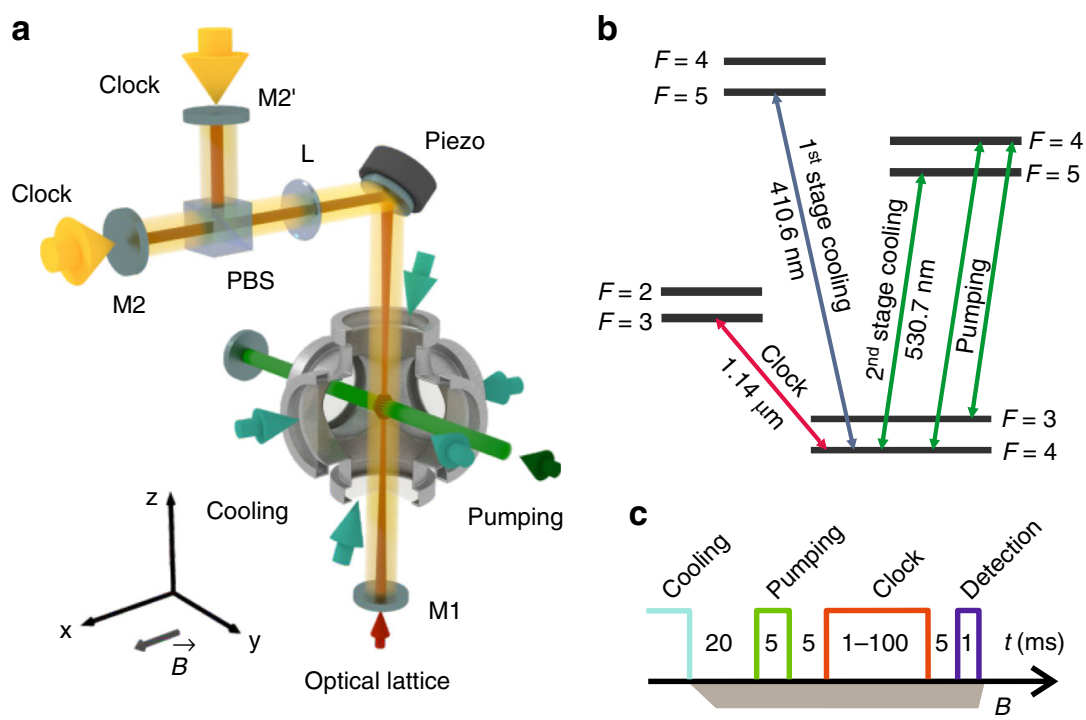

Fig. 1 Experimental details. a Schematic of the setup: the enhancement cavity forming the vertical optical lattice is built in a $\Gamma$-shape configuration with an intracavity polarizing beam splitter (PBS). For different polarizations, the two output couplers $M 2$ and $M 2^{\prime}$ are used. The clock laser radiation at $1.14 \mu \mathrm{m}$ is fed collinearly with the cavity axis through one of the cavity outcouplers with corresponding polarization; the pumping and repumping laser beams form standing waves along the $y$-axis; and the initial position of the Tm magneto-optical trap is adjusted to the cavity waist. M1: cavity incoupler, L: lens. b Relevant Tm level scheme. c Pulse sequence for spectroscopy of the clock transition. Two-stage laser cooling is followed by pumping to the $\mid F=4$, $\left.m_{\mathrm{F}}=0\right\rangle$ ground state using $530.7 \mathrm{~nm}$ radiation. The optical lattice is continuously on. After excitation of the clock transition, one measures the fluorescence of atoms remaining in the ground state by a $1 \mathrm{~ms}$ pulse (detection) at $410.6 \mathrm{~nm}$ (the same transition as for the first-stage cooling). The bias magnetic field $B$ is denoted by a gray bar on the bottom 
pulses at $530.7 \mathrm{~nm}$ in the presence of a bias magnetic field of $B_{x} \sim 0.1 \mathrm{G}$. After the optical pumping cycle, $10^{5}$ atoms are trapped in the lattice, with more than $80 \%$ in the target state. To interrogate the clock transition, we use radiation of a semiconductor $1.14 \mu \mathrm{m}$ laser stabilized to a high-finesse ultralowexpansion glass (ULE) cavity, providing a relative frequency instability of smaller than $10^{-14}$ over $1-100 \mathrm{~s}$ integration time $\mathrm{e}^{36}$. The linear frequency drift of $29 \mathrm{mHz} \mathrm{s}^{-1}$ is compensated using an acousto-optical modulator. After compensation, the clock laser can be used as a stable frequency reference for studying frequency shifts of the clock transition.

Magic wavelength determination. The important step toward high-resolution spectroscopy of the clock transition is the determination of the magic wavelength of the optical lattice when the dynamic polarizabilitites of the clock levels become equal ${ }^{37}$. We numerically calculated the dynamic polarizabilities of the clock levels using time-dependent second-order perturbation theory and transition data obtained using the COWAN package ${ }^{38}$ (Model 1 is described in Methods and in ref. ${ }^{34}$ ), and we predicted the existence of the magic wavelength at $811.2 \mathrm{~nm}$ (for the collinear magnetic field $\mathbf{B}$ and the lattice field polarization $\boldsymbol{\varepsilon}$ ) near a narrow transition from the upper $J=5 / 2$ clock level at $809.5 \mathrm{~nm}$. The calculated differential polarizability is shown in Fig. 2 with the red solid line. This wavelength region is readily accessible by Ti:sapphire or powerful semiconductor lasers.

Using the approach described in ref. ${ }^{39}$, we experimentally searched for the magic wavelength for the clock transition in the spectral region of $810-815 \mathrm{~nm}$. The transition frequency shift $\Delta v$ as a function of optical lattice power $P$ was measured at different lattice wavelengths (see Methods). The differential dynamic polarizability $\Delta \alpha$ between the clock levels was calculated using the expression

$$
\Delta \alpha=-\frac{h c w^{2}}{16 a_{0}^{3} \eta} \frac{\Delta \nu}{P}
$$

In the paper, we use atomic units for polarizability, namely, 1 a.u. $=4 \pi \varepsilon_{0} a_{0}^{3}$. Here, $h$ is the Plank constant, $c$ is the speed of light, $a_{0}$ is the Bohr radius, and $w=126.0(2.5) \mu \mathrm{m}$ is the lattice beam radius as calculated from the enhancement cavity geometry.

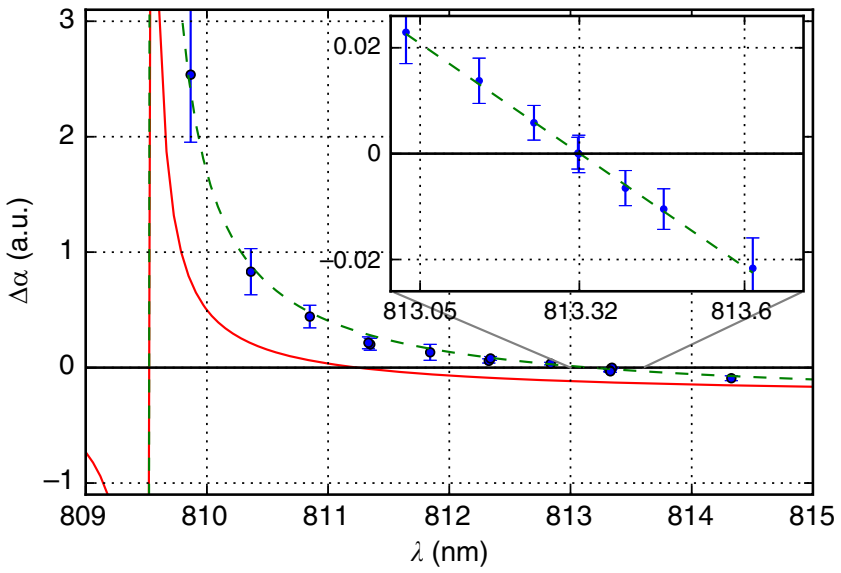

Fig. 2 Magic wavelength determination. Calculated (Model 1, red solid curve), measured (blue dots), and fitted (Model 2, green dashed line) differential dynamic polarizability $\Delta \alpha$ between the upper $(J=5 / 2)$ and the lower $(J=7 /$ 2) clock levels; theoretical models are further described in the main text, including the Methods section. Inset: magnified view of the spectral region around the magic wavelength $\lambda_{\mathrm{m}}=813.320(6) \mathrm{nm}$. Error bars represent $1 \mathrm{s.d}$. (standard deviation) of the measured polarizability values
The intracavity power $P$ was determined by calibrated photodiodes placed after the cavity outcouplers $M 2$ and $M 2$ '. For a specified beam radius $w$ and ground state dynamic polarizability $\alpha=195$ a.u. at $\lambda=813 \mathrm{~nm}^{34}$, the lattice depth for $P=1 \mathrm{~W}$ is $U=82 E_{\mathrm{r}}$, where $E_{\mathrm{r}}=h \times 1785 \mathrm{~Hz}$ is the recoil energy. Coefficient $\eta \leq 1$ accounts for the decrease of the average intensity for an atom with a non-zero kinetic energy. The details of the beam waist determination, power measurements, and coefficient $\eta$ calculations are given in the Methods section. Figure 2 shows the spectral dependency of $\Delta \alpha$. The magic wavelength of $\lambda_{\mathrm{m}}=$ $813.320(6) \mathrm{nm}$ was determined by the zero crossing of the linear fit in the inset of Fig. 2.

Trapping Tm atoms in the optical lattice at $\lambda_{\mathrm{m}}$ drastically reduces inhomogeneous ac Stark broadening of the clock transition. Using excitation with a $80 \mathrm{~ms}$-long Rabi $\pi$-pulses of the clock laser, we recorded a spectrum with $10 \mathrm{~Hz}$ full width at the half maximum shown in Fig. 3a. The nonunity excitation at the line center comes from a nonperfect initial polarization of the atoms and finite lifetime of the upper clock level ( $\tau=112 \mathrm{~ms}$ ).

Using a Ti:sapphire frequency comb, we determined the absolute frequency of the $|J=7 / 2, F=4\rangle \rightarrow|J=5 / 2, F=3\rangle$ clock transition in $\mathrm{Tm}$ of $262^{\prime} 954^{\prime} 938^{\prime} 269^{\prime} 213(30) \mathrm{Hz}$. The relative frequency uncertainty of $1.1 \times 10^{-13}$ comes mainly from the instability and calibration accuracy of a GLONASS-calibrated passive hydrogen maser used as a frequency reference for the comb.

Differential polarizability analysis. In the second-order approximation, the energy shift of an atomic level $\left|J, F, m_{\mathrm{F}}\right\rangle$ in an external oscillating electromagnetic field with wavelength $\lambda$ equals $-\alpha_{J, F, m_{\mathrm{F}}}(\lambda) E^{2} / 4$, where $E$ is the amplitude of the electric field. For linear field polarization, the dynamic polarizability $\alpha_{J, F, m_{\mathrm{F}}}$ can be split into scalar $\alpha_{J}^{\mathrm{s}}$ and the tensor $\alpha_{J, F}^{\mathrm{t}}$ parts as follows:

$$
\alpha_{J, F, m_{\mathrm{F}}}=\alpha_{J}^{\mathrm{S}}+\frac{3 \cos ^{2} \Theta-1}{2} \times \frac{3 m_{\mathrm{F}}^{2}-F(F+1)}{F(2 F-1)} \alpha_{J, F}^{\mathrm{t}},
$$

where $\Theta$ is the angle between the quantization axis (here, the direction of the external magnetic field B) and the electric field polarization $\varepsilon$ of the optical lattice. In our case, the differential polarizability of the two clock levels equals

$$
\Delta \alpha \equiv \alpha_{5 / 2,3,0}-\alpha_{7 / 2,4,0}=\Delta \alpha^{\mathrm{s}}+\frac{3 \cos ^{2} \Theta-1}{2} \Delta \alpha^{\mathrm{t}},
$$

where $\Delta \alpha^{\mathrm{s}}=\alpha_{5 / 2}^{\mathrm{s}}-\alpha_{7 / 2}^{\mathrm{s}}$ and $\Delta \alpha^{\mathrm{t}}=\frac{5}{7} \alpha_{7 / 2,4}^{\mathrm{t}}-\frac{4}{5} \alpha_{5 / 2,3}^{\mathrm{t}}$. By definition, at the magic wavelength $\lambda_{\mathrm{m}}$ the differential polarizability vanishes: $\Delta \alpha\left(\lambda_{\mathrm{m}}\right)=0$.

The frequency shift of the clock transition due to the optical lattice can be caused by (i) inaccuracy of the magic wavelength determination and (ii) angular dependency of the tensor part of the differential polarizability. The accuracy of the magic wavelength determination is related to the slope of $\Delta \alpha(\lambda)$ in the vicinity of $\lambda_{\mathrm{m}}$, which is $-0.075(17)$ a.u $\mathrm{nm}^{-1}$ in Tm, as shown in the inset in Fig. 2. In dimensionless normalized units this corresponds to $\frac{\delta(\Delta \alpha) / \alpha}{h \delta \nu_{\mathrm{L}} / E_{\mathrm{r}}} \approx 1.5 \times 10^{-12}$ (here $\delta v_{\mathrm{L}}$ is a lattice laser frequency increment), that is more than one order of magnitude smaller than the corresponding sensitivity of $\mathrm{Sr}$ and $\mathrm{Yb}$ lattice clocks 39,40

For $\Theta \ll 1$, the $\Theta$-dependent part of the differential tensor polarizability $\Delta \alpha^{\mathrm{t}}$ influences the clock transition frequency as follows:

$$
h \Delta \nu \approx 3 / 2 \Delta \alpha^{\mathrm{t}} \frac{E^{2}}{4} \Theta^{2}
$$



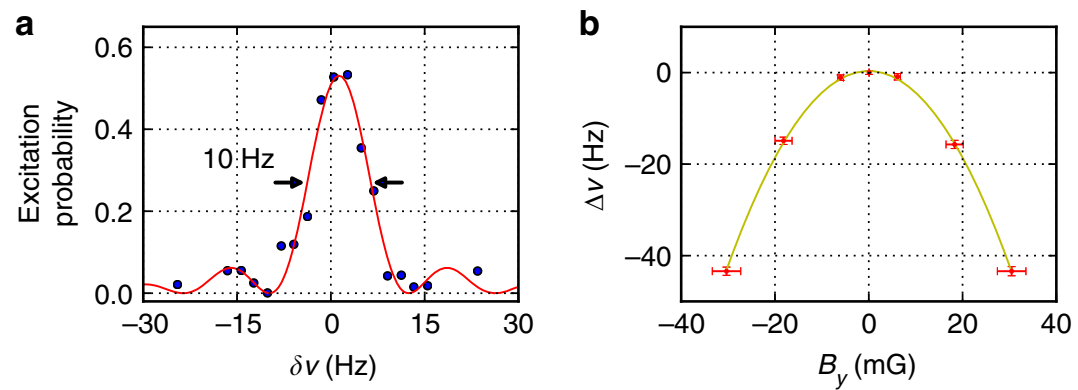

Fig. 3 Spectroscopy of the clock transition. a Spectral line shape of the clock transition in Tm. Every point is an average of six measurements. The solid curve shows the fit calculated for a Fourier-limited $80 \mathrm{~ms}$ rectangular $\pi$-pulse. $\delta \nu$ is the detuning from an exact resonance. $\mathbf{b}$ Clock transition frequency shift $\Delta \nu$ depending on $B_{y}$ (dots) at $B_{z}=0$ and constant $B_{x}=225 \mathrm{mG}$; the solid line is a parabolic fit. The dependence on $B_{z}$ is similar. Error bars represent $1 \mathrm{~s}$.d. of the $B_{y}$ magnetic field (horizontal) and of the measured frequency shift (vertical)
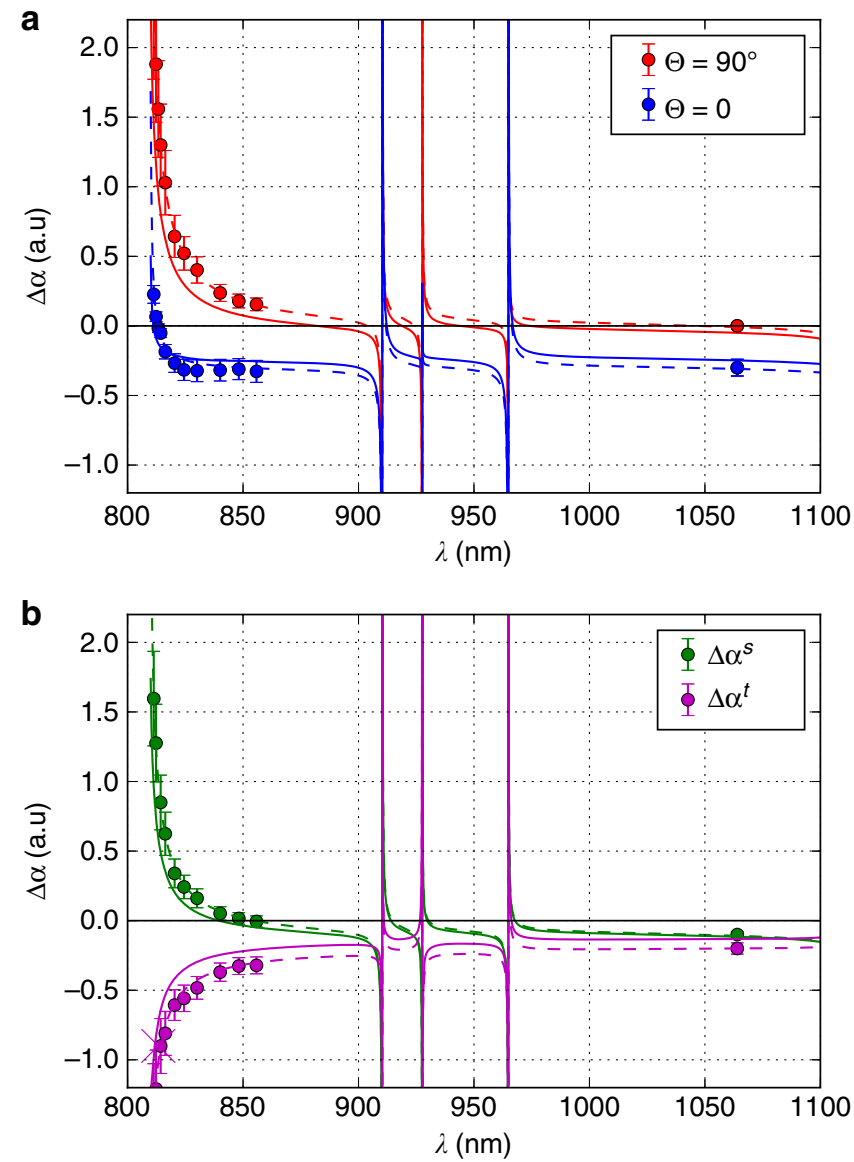

Fig. 4 Differential polarizability spectra. a Differential dynamic polarizability $\Delta \alpha(\lambda)$ for $\Theta=\pi / 2$ (red dots) and $\Theta=0$ (blue dots). b Corresponding scalar $\Delta \alpha^{\mathrm{s}}(\lambda)$ (green dots) and tensor $\Delta \alpha^{\mathrm{t}}(\lambda)$ (magenta dots) parts. The magenta cross is $\Delta \alpha^{t}\left(\lambda_{m}\right)$ determined from measurements in Fig. 3b. The solid and dotted curves are calculations based on Models 1 and 2, respectively (see Methods section). Error bars represent $1 \mathrm{~s}$.d. of the measured polarizability values

To find $\Delta \alpha^{\mathrm{t}}$, we measured the dependency of the clock transition frequency shift $\Delta v$ for a small magnetic field $B_{y}$ using the constant-bias fields $B_{x}=225 \mathrm{mG}$ and $B_{\mathrm{z}}=0\left(\Theta \approx B_{y} / B_{x}\right)$ as shown in Fig. 3b. From the corresponding parabolic coefficient of $-56(11) \mathrm{mHz} \mathrm{mG}^{-2}$, we obtained a differential tensor polarizability of $\Delta \alpha^{\mathrm{t}}=-0.9(2)$ a.u. at $\lambda_{\mathrm{m}}$. The uncertainty comes from the absolute calibration of the magnetic field and power calibration (see the Methods section). Both lattice frequency

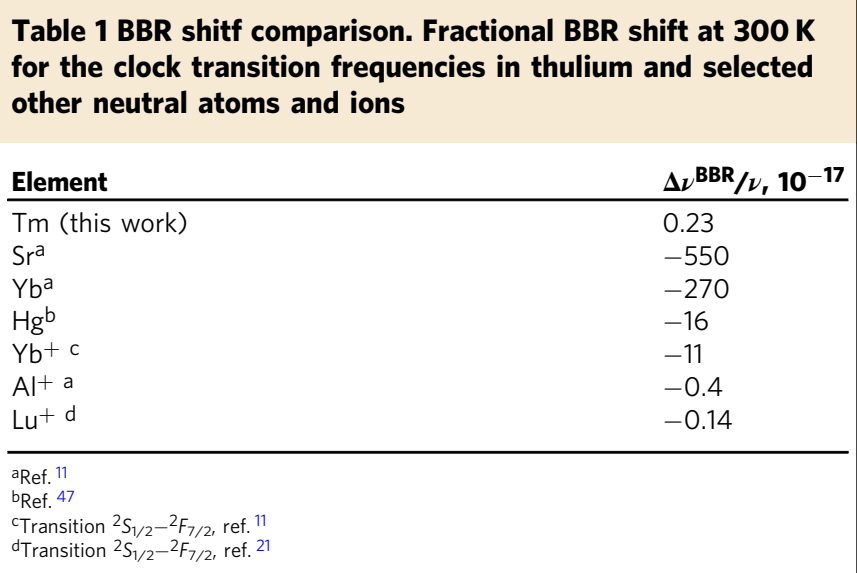

shifts mentioned in the previous paragraph can be readily reduced to the $\mathrm{mHz}$ level by stabilizing the lattice wavelength with $0.1 \mathrm{GHz}$ accuracy and by maintaining $|\Theta|<10^{-3}$.

Static differential polarizability and the BBR shift. The BBR shift of the clock transition frequency can be accurately calculated from the static differential scalar polarizability $\Delta a_{\mathrm{DC}}^{\mathrm{s}}=\Delta a^{\mathrm{s}}(\lambda \rightarrow \infty)$ using a theoretical model based on the measured polarizability spectrum at the wavelengths of $810-860 \mathrm{~nm}$ and at $1064 \mathrm{~nm}$.

Measurements in the spectral region of $810-860 \mathrm{~nm}$ were done by scanning the wavelength of the Ti:sapphire laser at two polarizations corresponding to $\Theta=0(\boldsymbol{\varepsilon}|| \mathbf{x})$ and $\Theta=\pi / 2(\boldsymbol{\varepsilon}|| \mathbf{y})$ as shown in Fig. 4a. The corresponding scalar $\Delta \alpha^{\mathrm{s}}(\lambda)$ and tensor $\Delta \alpha^{\mathrm{t}}$ $(\lambda)$ differential polarizabilities calculated from Eq. (3) are shown in Fig. $4 b$.

To measure the dynamic polarizability at $1064 \mathrm{~nm}$, we used a slightly different procedure: Tm atoms were trapped in the optical lattice at $\lambda_{\mathrm{m}}$ for which the differential polarizability vanishes, and the atomic cloud was illuminated along the $y$-axis by a focused beam of a linearly polarized single-frequency $1064 \mathrm{~nm}$ fiber laser with an optical power of up to $10 \mathrm{~W}$. The corresponding results for $\Theta=0(\boldsymbol{\varepsilon}|| \mathbf{x})$ and $\Theta=\pi / 2(\boldsymbol{\varepsilon} \| \mathbf{z})$ are also shown in Fig. 4.

To compare with experimental data and to deduce $\Delta a_{\mathrm{DC}}^{\mathrm{s}}$, we use Model 2 (see Methods), which differs from Model 1 by introducing four adjustable parameters: probabilities of the 806.7 and $809.5 \mathrm{~nm}$ transitions and two offsets for differential scalar and tensor polarizabilities. Corresponding fits based on Model 2 are shown as dashed lines in Fig. 4, while calculations with no free parameters (Model 1) are shown as solid lines. From Model 2, 
we obtain

$$
\Delta \alpha_{\mathrm{DC}}^{\mathrm{s}}=-0.063(30) \text { a.u. }
$$

Note, that the differential static scalar polarizability from Model 1 is -0.062 a.u. The BBR frequency shift can be readily calculated using the value of $\Delta a_{\mathrm{DC}}^{\mathrm{s}}{ }^{34}$. The differential scalar polarizability in the spectral region around $10 \mu \mathrm{m}$ (the maximum of the BBR spectrum at room temperature) differs by $<10^{-3}$ a.u. from $\Delta a_{\mathrm{DC}}^{\mathrm{s}}$. Note that there are no transitions from the clock levels for $\lambda>1.5 \mu \mathrm{m}$. For the clock transition at $1.14 \mu \mathrm{m}$, the room temperature BBR shift is $0.60(28) \mathrm{mHz}$, which is a few orders of magnitude smaller than that for other neutral atoms and is comparable to that of the best ion species as shown in Table 1. This result quantitatively confirms the idea of strong shielding of inner-shell transitions in lanthanides from external electric fields.

\section{Discussion}

The specific shielding of the inner-shell magnetic-dipole clock transition in atomic thulium at $1.14 \mu \mathrm{m}$ by the outer $5 s^{2}$ and $6 s^{2}$ electronic shells results in a very low sensitivity of its frequency to external electric fields. The differential static scalar polarizability of the two $J=7 / 2$ and $J=5 / 2$ clock levels is only $-0.063(30)$ atomic units, which corresponds to the fractional BBR frequency shift of the transition of $2.3(1.1) \times 10^{-18}$ at room temperature. This amount is three orders of magnitude less than that for the prominent clock transitions in $\mathrm{Sr}$ and $\mathrm{Yb}$ (see Table 1). Unlike other systems used for optical clocks, the clock states in thulium have a large orbital momentum, which leads to tensor light shifts. Additionally, the presence of the hyperfine splitting results in the second-order Zeeman shift. Our estimations of these and other sources of the clock transition frequency shift are summarized in the Methods section and show that they can be routinely controlled to better than $10^{-17}$. Altogether, this development makes Tm a promising candidate for a transportable room-temperature optical atomic clock with $10^{-17}$ uncertainty due to soft constraints on the ambient temperature stability. This approach combines advantages of unprecedented frequency stability of optical lattice clocks on neutral atoms and low sensitivity to BBR of ion optical clocks. Moreover, precision spectroscopy in Tm opens possibilities for sensitive tests of Lorentz invariance ${ }^{5}$ and for a search of the fine structure constant variation ${ }^{41}$.

Optical clocks based on a $f-f$ transition in some other lanthanides with spinless nuclei could be even more attractive featuring the low sensitivity to magnetic fields due to the absence of the hyperfine structure and small BBR shift. For example, the fine-structure clock transition at the telecom-wavelength of 1.44 $\mu \mathrm{m}$ in laser-cooled erbium atoms $\mathrm{s}^{32,42}$ can be particularly interesting for optical frequency dissemination over fiber networks ${ }^{43}$.

\section{Methods}

Enhancement cavity. The optical lattice is formed inside a $\Gamma$-shaped enhancement cavity, as shown in Fig. 1a. The reflectivity of the curved (radius of curvature $r=$ $-250 \mathrm{~mm}$ ) incoupler mirror M1 equals $87 \%$ and matches losses introduced by the vacuum chamber viewports. Outcouplers $\mathrm{M} 2$ or $\mathrm{M} 2{ }^{\prime}$ are identical flat mirrors with reflectivity $R>99 \%$. The $45^{\circ}$ cavity mirror is mounted on a piezo actuator to lock to the laser frequency. The intracavity polarization is defined by a broadband polarization beam splitter; depending on the polarization, either the M2 or M2' outcoupler mirror is used. The intracavity lens has a focal length of $f=400 \mathrm{~mm}$.

Depending on the experimental geometry $(\Theta=0$ or $\Theta=\pi / 2)$, we couple corresponding linearly polarized radiation from the Ti:sapphire laser through the incoupler mirror M1. Intracavity polarization filtering by PBS defines the polarization angle and significantly improves the polarization purity of the optical lattice. The angle between the laser field polarization and the bias magnetic field is adjusted with accuracy better than $1^{\circ}$.

Measurement of differential dynamic polarizabilities. The differential polariz ability $\Delta \alpha$ of the clock levels is determined from the frequency shift of the corresponding transition $\Delta v$, circulating power $P$, and $\mathrm{TEM}_{00}$ cavity mode radius $w$ at

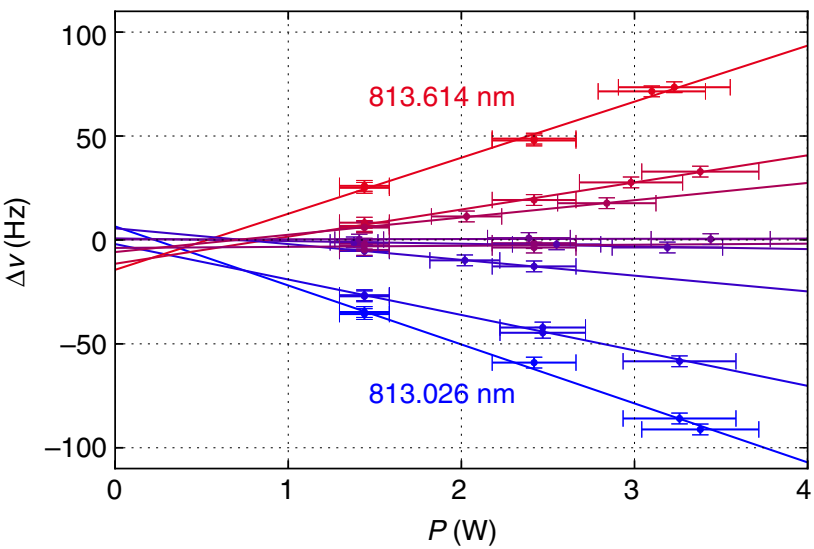

Fig. 5 Clock transition frequency shift measurements. Clock transition frequency shift $\Delta \nu$ as a function of optical lattice power $P$ in the vicinity of the magic wavelength $\lambda_{\mathrm{m}}=813.32 \mathrm{~nm}$. The solid lines are linear fits to the experimental data. The horizontal error bars correspond to uncertainty (1 s.d.) of the power measurements, and the vertical error bars are the mean squared transition line center fit errors and $2.6 \mathrm{~Hz}(1$ s.d. of the clock laser output frequency)

the atomic cloud position through Eq. (1). The dependency $\Delta v(P)$ was obtained for different wavelengths in the spectral range of $810-860 \mathrm{~nm}$ for intracavity circulating powers varying from 1 to $4 \mathrm{~W}$, as shown in Fig. 5 . The frequency shift $\Delta v$ was measured relative to the laser frequency, which is stabilized to an ultrastable ULE cavity with linear drift compensation. The slope coefficients of the corresponding linear fits were substituted into Eq. (1) to deduce $\Delta \alpha$ as presented in Figs. 2 and 4. Some residual nonlinearity and, as a consequence, imperfect intersection of linear fits at $P=0$ can be associated with finite atomic temperature which is discussed below. Note that differential polarizabilities obtained from linear fits with an additional constraint $\Delta v(0)=0$ agree with values obtained from our current analysis within one standard deviation.

Coefficient $\eta$ accounts for the effect of the reduced average light intensity $I_{\text {av }}=$ $\eta I_{0}$ due to the finite temperature of the atomic cloud in the $1 \mathrm{D}$ optical lattice with an intensity profile $I(r, z)=I_{0} \mathrm{e}^{-2 r^{2} / w^{2}} \cos ^{2}\left(2 \pi z / \lambda_{\mathrm{m}}\right)$. Assuming a Boltzmann distribution of the atoms with a temperature $T$ in the trap of depth $U_{0}=100$.. $300 E_{\mathrm{r}}$ (experimental lattice depth range), we estimate the coefficient $\eta$ using the following expression:

$$
\eta=\sum_{n_{z}} \frac{\mathrm{e}^{-E_{n_{z}} / k T}}{Z_{0}} \eta_{z}\left(n_{z}\right) \eta_{r}\left(n_{z}\right),
$$

Here the first multiplier gives the relative population of the $n_{z}$ axial vibrational mode with the energy $E_{n_{z}}$ (a number of axial vibrational states varies from 7 to 11 for $\left.U_{0}=100 \ldots 300 E_{\mathrm{r}}\right), \eta_{z}\left(n_{z}\right)$ and $\eta_{r}\left(n_{z}\right)$ are the coefficients due to delocalization in the axial and the radial directions, correspondingly. To estimate $\eta_{z}\left(n_{z}\right)$ we used numerically calculated wave functions of the axial vibrational modes in a singlewell sinusoidal potential. $\eta_{r}\left(n_{z}\right)$ was calculated using classical approximation:

$$
\eta_{r}\left(n_{z}\right)=\frac{\int_{0}^{U_{0}-E_{n_{z}}} \mathrm{e}^{-E / k T}\left(\frac{1}{2 r_{0}} \int_{-r_{0}}^{r_{0}} \mathrm{e}^{-2 r^{2}} \mathrm{~d} r\right) \mathrm{d} E}{\int_{0}^{U_{0}-E_{n_{z}}} \mathrm{e}^{-E / k T} \mathrm{~d} E},
$$

where $r_{0}=\left(-\ln \left(1-E / U_{0}\right) / 2\right)^{-1 / 2}$ is the classical turning point. We estimate parameter $\eta$ to be $0.76(15)$ for $k T=0.3 U_{0}$, which is close to an expected atomic temperature in our experiment. The stated error includes both the uncertainty of the atomic temperature, as well as incompleteness of the model (the single-well sinusoidal potential instead of the optical lattice potential and a possible difference in radial and axial temperatures).

The uncertainty of the frequency shift $\Delta v$ comes from the residual instability of the reference cavity on time intervals of $1000 \mathrm{~s}$. To estimate this uncertainty, we measured the clock transition frequency relative to the clock laser frequency at the magic wavelength where the perturbations from the lattice are minimal. The results are shown in Fig. 6 . The standard deviation equals $2.6 \mathrm{~Hz}$, contributing 0.003 a.u. to the error budget of $\Delta \alpha$. For the lattice wavelength detuned from $\lambda_{\mathrm{m}}$, the contribution of the laser frequency instability is negligible.

The intracavity power $P$ was determined by measuring power leaking through the cavity outcoupler M2 (or M2') using calibrated photodiodes. For each photodiode, we measured the power-to-voltage transfer function $P(U)=\kappa U$, where $U$ is the voltage reading from the photodiode and $\kappa$ is the coefficient measured using an absolutely calibrated Thorlabs S121C power meter. To determine $\kappa$, we unlocked the cavity, slightly tilted the outcoupler, blocked the reflected beam to 


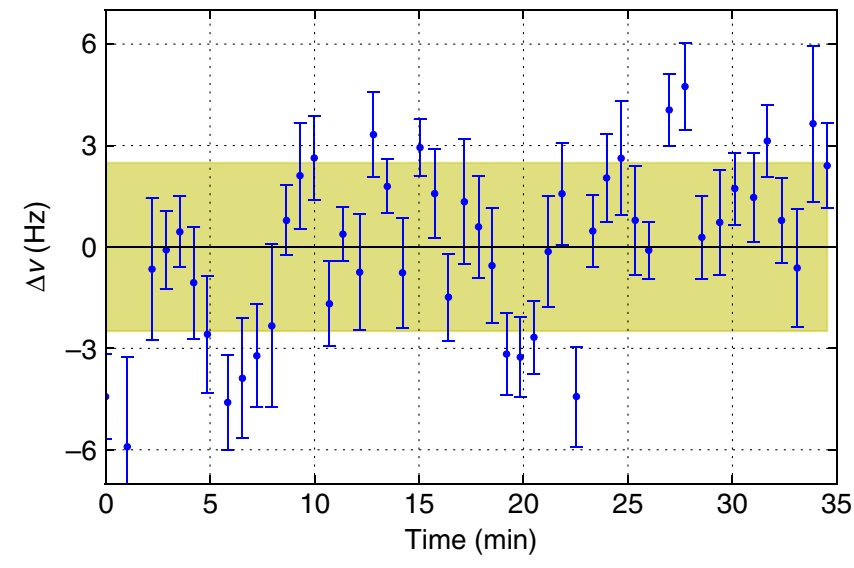

Fig. 6 Stability of the clock laser frequency. The relative frequency of the Tm clock transition and ULE cavity mode with linear drift compensation. Each data point and corresponding uncertainty comes from the fit of the clock transition spectrum. The shaded region corresponds to $1 \mathrm{~s}$.d. of the data set

prevent feasible reflections from the incoupler, and measured the power before the outcoupler and corresponding voltage reading of the photodiode. The linearity of the photodiode response was checked separately and was found to be better than $3 \%$ in the working region. The photodiode calibration was done in the whole spectral range of $810-860 \mathrm{~nm}$ to take into account the spectral response of the outcoupler and the photodiode. Although the specified uncertainty of the power sensor Thorlabs S121C equals 3\%, we estimate the net uncertainty of power measurement to be $10 \%$ based on a comparison of readings from three different absolutely calibrated sensors.

The beam radius $w$ at the atomic cloud position is deduced from the cavity geometry: distances from the vacuum chamber center (and target atomic cloud position) to M1, L and M2 (or M2') are 244,384 , and $500 \mathrm{~mm}$, respectively, giving a beam radius of $w=126 \mu \mathrm{m}$ at the position of atomic cloud. The uncertainty of $w$ comes from the position uncertainties of cavity elements and of the atomic cloud with respect to the chamber center, in addition to the uncertainty of $1 \mathrm{~mm}$ of the lens focal length. We conservatively evaluate the position uncertainties of M1, L, and M2 (or PBS and $\mathrm{M}^{\prime}$ ) as $1 \mathrm{~mm}$ and the possible axial displacement of the atomic cloud of $2 \mathrm{~mm}$. The partial contributions to the beam radius uncertainty are $1.6 \mu \mathrm{m}$ (the incoupler), $1.2 \mu \mathrm{m}$ (the lens), $0.03 \mu \mathrm{m}$ (the outcoupler), $0.3 \mu \mathrm{m}$ (the cloud), and $1.5 \mu \mathrm{m}$ (focal length). Adding up in quadratures, the total uncertainty of the beam radius $w_{0}$ equals $2.5 \mu \mathrm{m}$. The result is independently confirmed by measuring frequency intervals between cavity transversal modes.

Summarizing, we evaluate the uncertainty of measured differential polarizability $\Delta \alpha$ to be $22 \%$ with the dominating contribution from the lattice intensity uncertainty. In the vicinity of $\lambda_{\mathrm{m}}$, the uncertainty is slightly higher by 0.003 a.u. because of the reference clock laser frequency instability.

To measure differential polarizability at $1064 \mathrm{~nm}$, atoms were trapped in the optical lattice at the magic wavelength and irradiated by focusing a slightly elliptic $1064 \mathrm{~nm}$ laser beam with waists of $w_{x}=320(20) \mu \mathrm{m}$ and $w_{y}=280(20) \mu \mathrm{m}$ in the $x$ and $y$ directions, respectively. To adjust the $1064 \mathrm{~nm}$ laser beam center to the atomic cloud, we maximized the intensity of the beam on the atomic cloud by monitoring the frequency shift of the clock transition. To increase the sensitivity, corresponding measurements were performed by tuning the clock laser to the slope of the $1.14 \mu \mathrm{m}$ transition.

For the measurement session of $\Delta \alpha$ at $\Theta=0$, we performed three adjustments of the $1064 \mathrm{~nm}$ laser beam. The reproducibility corresponded to a frequency shift of $5 \mathrm{~Hz}$ at the maximum frequency shift of $25 \mathrm{~Hz}$. The resulting linear coefficient was evaluated as $\Delta v / P=3.1(6) \mathrm{Hz} \mathrm{W}^{-1}$ including this uncertainty. In the $\Theta=\pi / 2$ configuration, we did not observe any significant effect from adjustment; the coefficient was $-0.04(24) \mathrm{Hz} \mathrm{W}^{-1}$.

Theoretical analysis. The theoretical approach to calculate the polarizabilities of $\mathrm{Tm}$ atomic levels was described in our previous works ${ }^{34,44}$. These calculations are based on the time-dependent second-order perturbation theory with summation over known discrete transitions from the levels of interest. For calculations, we used transitions wavelengths and probabilities obtained with the COWAN package ${ }^{38}$ with some exceptions: for transitions with $\lambda>800 \mathrm{~nm}$, experimental wavelengths from ref. ${ }^{45}$ are used. This approach allows the accuracy of the magic wavelength prediction to be increased in the corresponding spectral region of $\lambda>800 \mathrm{~nm}$. According to calculations, the magic wavelength was expected at $811.2 \mathrm{~nm}$, which motivated our experimental studies in the spectral region $810-815 \mathrm{~nm}$ (see Fig. 2). We refer to this model as Model 1 and use it for comparison with the experimental results of this work as shown in Figs. 2 and 4.

The deviation of the experimental data from Model 1 can be explained by two main factors. First, we did not take into account transitions to the continuum in this model. Together with uncertainties of COWAN calculations of transition amplitudes, this aspect can result in a small offset of the infrared differential polarizability spectrum. Note that although transitions to the continuum spectrum may significantly contribute to polarizabilities of the individual levels (up to $10 \%$ ), for the differential polarizability of the $f-f$ transition at $1.14 \mu \mathrm{m}$ in the Tm contribution of the continuum is mostly canceled ${ }^{34}$.

To fit experimental data, we use Model 2, which differs from Model 1 through the introduction of four fitting parameters. For these parameters, we use the probabilities of the 806.7 and $809.5 \mathrm{~nm}$ transitions, which mostly affect the polarizability spectrum in the $810-860 \mathrm{~nm}$ region and the two offsets for the scalar and tensor polarizabilities. After fitting the experimental data (see Fig. 4) by Model 2 , the probability of the $806.7 \mathrm{~nm}$ transition is changed from 3473 to $4985(1100)$ $\mathrm{s}^{-1}$, the probability of the $809.5 \mathrm{~nm}$ transition is changed from 149 to $424(70) \mathrm{s}^{-1}$, and the fitted offsets for the differential scalar and tensor polarizabilities equal $-0.006(30)$ and $-0.074(30)$ a.u.

The transitions from the upper Tm clock level $J=5 / 2$ in the spectral range $\lambda>$ $900 \mathrm{~nm}$ are weak, and their probabilities are not experimentally measured. To calculate probabilities, we used the COWAN package. To estimate the impact of insufficient knowledge of the transition probabilities on the differential scalar polarizability $\Delta \alpha^{s}(\lambda)$, we assume a potential variation of each transition probability by a factor of 2 . After extrapolation of the fitted Model 2 to $\lambda \rightarrow \infty$, we obtain the static differential polarizability $\Delta \alpha_{\mathrm{DC}}^{\mathrm{s}}=-0.063_{-0.005}^{+0.01}$ a.u., where the uncertainty comes from the variation of the $\lambda>900 \mathrm{~nm}$ transition probabilities.

We summarize all sources of uncertainty that contribute to the error of the static differential polarizability $\Delta a_{\mathrm{DC}}^{\mathrm{s}}$ in Table 2 . As discussed above, the experimental uncertainty for the $810-860 \mathrm{~nm}$ range contributes 0.025 a.u. to the $\Delta a_{\mathrm{DC}}^{\mathrm{s}}$, while the error in the measurement at $1064 \mathrm{~nm}$ results in 0.014 a.u. variation of Model 2 extrapolation. The uncertainty of the angle $\Theta$ adjustment contributes 0.002 a.u. The uncertainty associated with the poorly known transition probabilities from the $J=5 / 2$ clock level in $\lambda>900 \mathrm{~nm}$ range contributes 0.01 a.u. Using extrapolation of Model 2 and adding all uncertainties, we obtain the final result of $\Delta a_{\mathrm{DC}}^{\mathrm{s}}=-0.063(30)$ a.u. Note that this finding is fully consistent with the extrapolated value of -0.062 a.u. obtained from Model 1 and given for the reference in Table 2.

Frequency uncertainty estimation. Here, we discuss expected sources of the 1.14 $\mu \mathrm{m}$ clock transition frequency shift and uncertainty. We evaluate necessary control over the experimental parameters to maintain corresponding uncertainty below $1 \mathrm{mHz}$, or $4 \times 10^{-18}$ in fractional units.

Zeeman shift: The frequency of the $\left|F=4, m_{\mathrm{F}}=0\right\rangle \rightarrow\left|F=3, m_{\mathrm{F}}=0\right\rangle$ clock transition possesses a quadratic sensitivity to a dc magnetic field $B$ with a coefficient $\beta=257.2 \mathrm{~Hz} \mathrm{G}^{-234}$. To provide uncertainty of the transition frequency below $1 \mathrm{mHz}$, it would be necessary to stabilize the magnetic field at the level of 20 $\mu \mathrm{G}$ at the bias field of $B=100 \mathrm{mG}$. However, the quadratic Zeeman shift in Tm can be fully canceled by measuring an averaged frequency of two clock transitions $\mid F=$ $\left.4, m_{\mathrm{F}}=0\right\rangle \rightarrow\left|F=3, m_{\mathrm{F}}=0\right\rangle$ and $\left|F=3, m_{\mathrm{F}}=0\right\rangle \rightarrow\left|F=2, m_{\mathrm{F}}=0\right\rangle$ (Fig. 1b) with the quadratic Zeeman coefficients of the opposite signs. The magic wavelength condition is simultaneously fulfilled for both transitions since $\alpha_{J,-1 / 2,0} \equiv \alpha_{J, J+1 / 2,0}$ for atoms with $I=1 / 2$ (index reads as $\left|J, F, m_{\mathrm{F}}\right\rangle$ ).

Lattice shift: The clock transition frequency shift due to the optical lattice was evaluated in the Differential polarizability analysis section. While the slope of the differential polarizability at the magic wavelength is less than that of the optical clock using other neutral atoms, the differential tensor polarizability of $0.9(2)$ a.u. imposes strict requirements on the quantization axis alignment and the lattice light polarization purity. Control of $|\Theta|<10^{-3}$ (which provides $\Delta v<1 \mathrm{mHz}$ according to Eq. (6) for a lattice depth of $100 E_{r}$ ) can be achieved through a calibration procedure similar to the one described in Fig. 3b. The magnetic field should be stabilized at the level $10^{-3} \mathrm{~B}$, or $0.1 \mathrm{mG}$ for the bias field of $100 \mathrm{mG}$.

Higher-order lattice shifts: Our estimations (using the method described in ref. ${ }^{34}$ ) show that the hyperpolarizability shift at $100 E r$ lattice depth should be $<10^{-17}$ in fractional units. Moreover, both hyperpolarizability and M1 and E2

\section{Table 2 Uncertainty budget for the differential scalar polarizability. All sources of uncertainty that contribute to the error of the static differential polarizability $\Delta a_{D C}^{s}$}

\section{Source}

Experimental results for $810-850 \mathrm{~nm}$

Experimental result for $1064 \mathrm{~nm}$

Angle $\Theta$

Transition probabilities for $\lambda>900 \mathrm{~nm}$

Total

For reference:

Difference between Models 1 and 2
Uncertainty (a.u.)

0.025

0.0014

0.002

0.01

0.030

0.001 
transition contribution shifts can be suppressed by reducing the trap depth or by using the operational wavelength ${ }^{40}$.

Since the electric field gradient in the optical lattice oscillates at the optical frequency, the time-averaged frequency shift associated with a permanent differential quadrupole moment of the clock transition is zero.

BBR electric field: The $1.14 \mu \mathrm{m}$ clock transition frequency shift due to the electric component of BBR at room temperature is $0.60(28) \mathrm{mHz}$, with uncertainty coming from the polarizability measurement error. Accordingly, even approximate stabilization of the ambient temperature would provide a clock performance of much better than $10^{-17}$.

BBR magnetic field: To estimate the clock transition frequency shift due to the magnetic component of BBR, we follow the analysis given in the work ${ }^{46}$. The corresponding frequency shift of one of the clock levels coupled to another atomic level with magnetic-dipole transition at frequency $\omega_{0}$ can be found by integrating over the full BBR spectrum as follows:

$$
\begin{aligned}
\Delta \nu_{\mathrm{bbr}}^{\mathrm{B}}(T) & =-\frac{\omega_{0}}{2 \pi} \frac{\mu_{\mathrm{B}}^{2}}{2 \hbar \pi^{2} c^{5} \varepsilon_{0}} \int_{0}^{\infty} \frac{1}{\omega_{0}^{2}-\omega^{2}} \frac{\omega^{3}}{e^{\hbar \omega / k T}-1} \mathrm{~d} \omega \\
& =-\frac{\omega_{0}}{2 \pi} \frac{\gamma}{2}\left(\frac{T}{T_{0}}\right)^{2} f(y),
\end{aligned}
$$

where $\varepsilon_{0}$ is the vacuum permittivity, $T_{0}=300 \mathrm{~K}$, and $y=\hbar \omega_{0} / k_{\mathrm{B}} T$. Here,

$$
\begin{gathered}
\gamma=\frac{\mu_{\mathrm{B}}^{2}}{\hbar^{2}} \frac{\hbar}{6 c^{5} \varepsilon_{0}}\left(\frac{k_{\mathrm{B}} T_{0}}{\hbar}\right)^{2} \approx 9.78 \times 10^{-18}, \\
f(y)=\frac{6}{\pi^{2}} \int_{0}^{\infty} \frac{1}{y^{2}-x^{2}} \frac{x^{3} \mathrm{~d} x}{e^{x}-1} .
\end{gathered}
$$

The hyperfine transition frequency $\omega_{0}$ of the $J=7 / 2$ ground level in Tm equals $2 \pi \times 1496 \mathrm{MHz}$, while for the $J=5 / 2$ clock level it equals $2 \pi \times 2115 \mathrm{MHz}$. For these values of $\omega_{0}, y \ll 1, f(y) \approx-1$, and the shift is on the order of $10^{-8} \mathrm{~Hz}$. To estimate the contribution from the optical transitions, we evaluate the shift from the lowest frequency magnetic-dipole transition at $\omega_{0} \approx 2 \pi \times 263 \mathrm{THz}$, which is the clock transition itself: $y=42, f(y)=2.3 \times 10^{-3}$, and $\Delta \nu_{\mathrm{bbr}}^{\mathrm{B}}\left(T_{0}\right)=-3 \times 10^{-6} \mathrm{~Hz}$ for the ground level (for the upper clock level, the corresponding shift is $+3 \times 10^{-6} \mathrm{~Hz}$ ). Hence, we estimate the total shift of the clock transition from the magnetic component of BBR to be $<10^{-4} \mathrm{~Hz}$, or $<10^{-18}$ in fractional units.

Stray electric fields: The differential static polarizability of the clock transition does not exceed 0.2 a.u. for any angle $\Theta$, which means that static stray fields of 1 $\mathrm{kV} \mathrm{m}^{-1}$ would produce a fractional frequency shift smaller than $1 \times 10^{-17}$. In turn, there is a differential quadrupole moment of clock levels (with an upper limit of $1 \mathrm{a}$. $\mathrm{u}^{34}$ ), which may lead to the static quadrupole frequency shift. Accordingly, one needs a stray electric field gradient of $5 \mathrm{kV} \mathrm{m}^{-2}$ to reach a fractional frequency shift of $1 \times 10^{-17}$. In regular lattice experiments, both stray electric fields and their gradients are much smaller than the abovementioned values.

\section{Data availability}

All data presented and analyzed in this study are available from the corresponding author upon reasonable request.

Received: 11 October 2018 Accepted: 20 March 2019

Published online: 12 April 2019

\section{References}

1. Chen, J. et al. Sympathetic ground state cooling and time-dilation shifts in an ${ }^{27} \mathrm{Al}^{+}$optical clock. Phys. Rev. Lett. 118, 053002 (2017).

2. Ushijima, I., Takamoto, M., Das, M., Ohkubo, T. \& Katori, H. Cryogenic optical lattice clocks. Nat. Photon. 9, 185-189 (2015).

3. Marti, G. E. et al. Imaging optical frequencies with $100 \mu \mathrm{Hz}$ precision and 1.1 $\mu \mathrm{m}$ resolution. Phys. Rev. Lett. 120, 103201 (2018)

4. Delva, P. et al. Test of special relativity using a fiber network of optical clocks. Phys. Rev. Lett. 118, 221102 (2017).

5. Shaniv, R. et al. New methods for testing lorentz invariance with atomic systems. Phys. Rev. Lett. 120, 103202 (2018).

6. Rosenband, T. et al. Frequency ratio of $\mathrm{Al}^{+}$and $\mathrm{Hg}^{+}$single-ion optical clocks; metrology at the 17th decimal place. Science 319, 1808-1812 (2008).

7. Borkowski, M. Optical lattice clocks with weakly bound molecules. Phys. Rev. Lett. 120, 083202 (2018).

8. Roberts, B. M. et al. Search for domain wall dark matter with atomic clocks on board global positioning system satellites. Nat. Commun. 8, 1195 (2017).

9. Wcisto, P. et al. New bounds on dark matter coupling from a global network of optical atomic clocks. Sci. Adv. 4, eaau4869 (2018)

10. Norcia, M. A. et al. Cavity-mediated collective spin-exchange interactions in a strontium superradiant laser. Science 361, 259-262 (2018).
11. Ludlow, A., Boyd, M., Ye, J., Peik, E. \& Schmidt, P. Optical atomic clocks. Rev. Mod. Phys. 87, 637 (2015)

12. Poli, N., Oates, C. W., Gill, P. \& Tino, G. M. Optical atomic clocks. Preprint at https://arxiv.org/abs/1401.2378 (2014).

13. Safronova, M. S. et al. Search for new physics with atoms and molecules. Rev. Mod. Phys. 90, 025008 (2018)

14. Koller, S. B. et al. Transportable optical lattice clock with $7 \times 10^{-17}$ uncertainty. Phys. Rev. Lett. 118, 073601 (2017).

15. Grotti, J. et al. Geodesy and metrology with a transportable optical clock. Nat. Phys. 14, 437-441 (2018).

16. Takano, T. et al. Geopotential measurements with synchronously linked optical lattice clocks. Nat. Photon. 10, 662-666 (2016).

17. Nicholson, T. et al. Systematic evaluation of an atomic clock at $2 \times 10^{-18}$ total uncertainty. Nat. Commun. 6, 6896 (2015).

18. Schioppo, M. et al. Ultrastable optical clock with two cold-atom ensembles. Nat. Photon. 11, 48-52 (2017).

19. Huntemann, N., Sanner, C., Lipphardt, B., Tamm, C. \& Peik, E. Single-ion atomic clock with $3 \times 10^{-18}$ systematic uncertainty. Phys. Rev. Lett. 116, 063001 (2016).

20. Chou, C., Hume, D., Koelemeij, J., Wineland, D. \& Rosenband, T. Frequency comparison of two high-accuracy $\mathrm{Al}^{+}$optical clocks. Phys. Rev. Lett. 104, 070802 (2010)

21. Arnold, K. J., Kaewuam, R., Roy, A., Tan, T. R. \& Barrett, M. D. Blackbody radiation shift assessment for a lutetium ion clock. Nat. Commun. 9, 1650 (2018).

22. Nauta, J. et al. Towards precision measurements on highly charged ions using a high harmonic generation frequency comb. Nucl. Instrum. Methods Phys. Res. Sect. B 408, 285-288 (2017).

23. Yu, Y.-m \& Sahoo, B. K. Selected highly charged ions as prospective candidates for optical clocks with quality factors larger than $10^{15}$. Phys. Rev. A 97, 041403 (2018)

24. Kozlov, M. G., Safronova, M. S., López-Urrutia, J. R. C. \& Schmidt, P. O. Highly charged ions: optical clocks and applications in fundamental physics. Rev. Mod. Phys. 90, 045005 (2018).

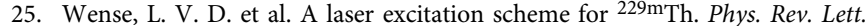
119, 132503 (2017).

26. Thielking, J. et al. Laser spectroscopic characterization of the nuclear-clock

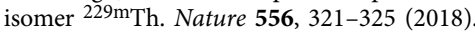

27. Aleksandrov, E. B., Vedenin, V. D. \& Kulyasov, V. N. Broadening and shift of thulium resonance lines by helium. Opt. Spectrosc. 56, 365-368 (1984).

28. Hancox, C. I., Doret, S. C., Hummon, M. T., Luo, L. \& Doyle, J. M. Magnetic trapping of rare-earth atoms at millikelvin temperatures. Nature 431, 281-284 (2004).

29. Hancox, C. I., Doret, S. C., Hummon, M. T., Krems, R. V. \& Doyle, J. M. Suppression of angular momentum transfer in cold collisions of transition metal atoms in ground states with nonzero orbital angular momentum. Phys. Rev. Lett. 94, 013201 (2005).

30. Probst, S. et al. Anisotropic rare-earth spin ensemble strongly coupled to a superconducting resonator. Phys. Rev. Lett. 110, 157001 (2013).

31. Dibos, A. M., Raha, M., Phenicie, C. M. \& Thompson, J. D. Atomic source of single photons in the telecom band. Phys. Rev. Lett. 120, 243601 (2018).

32. Kozlov, A., Dzuba, V. A. \& Flambaum, V. V. Prospects of building optical atomic clocks using Er i or Er iii. Phys. Rev. A 88, 032509 (2013).

33. Kozlov, A., Dzuba, V. A. \& Flambaum, V. V. Optical atomic clocks with suppressed blackbody-radiation shift. Phys. Rev. A 90, 042505 (2014).

34. Sukachev, D. et al. Inner-shell magnetic dipole transition in Tm atoms: a candidate for optical lattice clocks. Phys. Rev. A 94, 022512 (2016).

35. Sukachev, D. D. et al. Secondary laser cooling and capturing of thulium atoms in traps. Quantum Electron. 44, 515-520 (2014).

36. Alnis, J., Matveev, A., Kolachevsky, N., Udem, T. \& Hänsch, T. W. Subhertz linewidth diode lasers by stabilization to vibrationally and thermally compensated ultralow-expansion glass Fabry-Pérot cavities. Phys. Rev. A 77, 053809 (2008)

37. Takamoto, M. \& Katori, H. Spectroscopy of the ${ }^{1} S_{0}-{ }^{3} P_{0}$ clock transition of ${ }^{87} \mathrm{Sr}$ in an optical lattice. Phys. Rev. Lett. 91, 223001 (2003).

38. Cowan, R. The Theory of Atomic Structure and Spectra, and Cowan Programs $R C N, R C N 2$, and RCG (University of California Press, Berkeley, CA, 1981).

39. Barber, Z. W. et al. Optical lattice induced light shifts in an $\mathrm{Yb}$ atomic clock. Phys. Rev. Lett. 100, 103002 (2008).

40. Brown, R. C. et al. Hyperpolarizability and operational magic wavelength in an optical lattice clock. Phys. Rev. Lett. 119, 253001 (2017)

41. Kolachevsky, N. N. High-precision laser spectroscopy of cold atoms and the search for the drift of the fine structure constant. Phys.-Usp. 51, 1180-1190 (2008). 
42. McClelland, J. J. \& Hanssen, J. L. Laser cooling without repumping: a magneto-optical trap for erbium atoms. Phys. Rev. Lett. 96, 143005 (2006).

43. Riehle, F. Optical clock networks. Nat. Photon. 11, 25-31 (2017).

44. Golovizin, A. et al. Methods for determining the polarisability of the fine structure levels in the ground state of the thulium atom. Quantum Electron. 47, 479-483 (2017).

45. Kramida, A., Ralchenko, Yu., Reader, J., and NIST ASD Team. NIST Atomic Spectra Database (ver. 5.5.6), [Online]. https://physics.nist.gov/asd (National Institute of Standards and Technology, Gaithersburg, MD, 2018). Accessed 21 Aug 2018.

46. Gan, H. et al. Oscillating magnetic field effects in high precision metrology. Phys. Rev. A 98, 032514 (2018)

47. Tyumenev, R. et al. Comparing a mercury optical lattice clock with microwave and optical frequency standards. New J. Phys. 18, 113002 (2016).

\section{Acknowledgements}

Development of the ultrastable clock laser is financially supported by RFBR grant no. 1629-11723. V.S. acknowledges financial support from RFBR grant no. 18-02-00628. A.G., E.F., D.T., K.K., and N.K. acknowledge support from RSF grant no. 19-12-00137. We are grateful to S. Kanorski and V. Belyaev for invaluable technical support and to S. Fedorov for assembling the frequency comb. We also thank M. Barrett for very useful discussions.

\section{Author contributions}

A.G., E.F. and D.T. carried out all measurements and analyzed the data; A.G. and D.S. performed the theoretical calculations; and A.G., D.S. and N.K. wrote the paper. K.K., V.S. and N.K. conceived and directed the project. All authors discussed the results and commented on the manuscript.

\section{Additional information}

Supplementary Information accompanies this paper at https://doi.org/10.1038/s41467019-09706-9.

Competing interests: The authors declare no competing interests.

Reprints and permission information is available online at http://npg.nature.com/ reprintsandpermissions/

Journal Peer review Information: Nature Communications thanks the anonymous reviewers for their contribution to the peer review of this work. Peer reviewer reports are available.

Publisher's note: Springer Nature remains neutral with regard to jurisdictional claims in published maps and institutional affiliations.

(c) (i) Open Access This article is licensed under a Creative Commons Attribution 4.0 International License, which permits use, sharing, adaptation, distribution and reproduction in any medium or format, as long as you give appropriate credit to the original author(s) and the source, provide a link to the Creative Commons license, and indicate if changes were made. The images or other third party material in this article are included in the article's Creative Commons license, unless indicated otherwise in a credit line to the material. If material is not included in the article's Creative Commons license and your intended use is not permitted by statutory regulation or exceeds the permitted use, you will need to obtain permission directly from the copyright holder. To view a copy of this license, visit http://creativecommons.org/ licenses/by/4.0/.

(C) The Author(s) 2019 